\title{
The Effect of a Combined Practice Course of Mental and Physical Practice with Systematic Increase in Contextual Interference on Learning a Kata Skill
}

\author{
${ }^{1}$ Fateme Mohammadhasani, ${ }^{1}$ Robabeh Rostami ${ }^{*}{ }^{1}$ Majid Chhardah Cheric \\ ${ }^{1}$ Department of Motor Behavior, Faculty of Sport Sciences, Shiraz University, Shiraz, Iran.
}

\begin{abstract}
Background. Although some research showed the benefits of a systematic increase in contextual interference (CI), it is not completely proved in young children and novices. Objectives. On the other hand, considering the recommendation of researchers about the advantages and the use of the mental practice, the aim of the present study was to combine mental practice with CI to improve motor performance in a kata skill. This study investigates the simultaneous effects of these two types of practice. Methods. One school in Shiraz city was chosen randomly. Among the entire group of students in this school, 36 sixth-grade elementary school students of age 12 were chosen randomly to participate in this study. After filling out MIQ questionnaire, participants took part in initial instruction sessions and then participated in the pretest. In the next stage, they were randomly placed in three groups: blocked physical practice (B), physical practice with a systematic increase in CI and mental practice + physical practice with a systematic increase in CI (M-CI). The participants practised for five sessions and five trials in each session. In the last training session, an acquisition test, a subsequent retention test (48 hours later), and transfer test in a completion condition were conducted. Results. The results showed a significant difference in the acquisition test in the CI and B contexts, and in the retention test and transfer tests, in the CI and M-CI contexts. Conclusion. Considering the results, it can be deduced that the systematic increase in the CI has the highest effectiveness. After that, the combined practice (M-CI) has long-term positive effects on performance and learning a kata skill.
\end{abstract}

KEY WORDS: Mental Practice, Physical Practice, Kata Skill, Systematic Increase, Contextual Interference.

\section{INTRODUCTION}

Planning the practice session is an important factor with regard to the needs of learners (1). In learning motor skills, one of the planning methods of the practice variability is a phenomenon called 'contextual interference' Contextual interference (CI) is defined as an interference in function and learning, which is caused by exercising one task in the context of other tasks. The resulting CI effect depends on tasks, age, or the way tasks are presented in the practice session (2). A high CI needs more focus on acquiring the skill and problem-solving, and increases learning through this process. So, in a random practice schedule, since the learner changes the task in every trial, more $\mathrm{CI}$ is created, a fact which is expected to increase the learning rate, although it provides no good immediate performance. Blocked practice, due to its lower interference, will have a better performance but is not accompanied by better

*. Corresponding Author:

Robabeh Rostami

E-mail: rostami@ shirazu.ac.ir 
transfer and learning (3). Recently, Porter (2008) propounded the idea of systematically increasing CI (4). Porter et al. (2010) showed that a gradual increase in CI in this way leads to superior performance in retention and transfer tests later (5). Porter and Saemi (2010) investigated the effect of a systematic increase in CI on three different basketball-related passes. The results of the immediate and delayed retention test showed that practising with gradual increases in CI resulted in a superior performance, compared to traditional blocked and random scheduling (67). Porter (2008) conducted two experiments investigating an alternative form of a practice schedule. Experiment 1 tested the hypothesis that practising variations of the same tasks with systematic increases in CI would lead to a superior performance when practising the same tasks in a blocked or random schedule. The results showed that participants who followed the increasing schedule generally performed better on a retention and transfer test when compared to participants practising the same tasks following blocked and random schedules. The purpose of Experiment 2 was to test if the learning benefits of an increasing schedule were limited to variations of the same task or if the benefits were generalized to tasks with different invariant features, thus being controlled by different generalized motor programmes (GMP). The results showed that participants who practised with gradual increases in CI generally performed better on a retention and transfer test compared to participants who practised with traditional blocked or random scheduling (4).

The elaborative-processing and forgettingreconstruction hypotheses are the two principal explanations for the CI effect. The elaborativeprocessing hypothesis holds that random practice forces the learner into more elaborate processing, such as intertask comparisons and the embellishment of task-relevant information (8-10). More elaborate information processing is thought to result in more comprehensive and readily retrievable memory traces. According to the elaborative-processing hypothesis, the interval prior to the next movement trial (i.e. intertrial interval) is a likely time during which intertask comparisons are made and the elaboration occurs. The forgettingreconstruction hypothesis suggests that a previously constructed action plan is more likely to be available in the working memory during blocked practice because the same task is repeated (11). Random practice, however, forces the learner to abandon the action plan constructed previously because he or she has to perform a different task in the next trial. The forgetting that ensues requires the learner to actively reconstruct the action plan the next time when the initial task is practised, resulting in a stronger memory representation of the tasks (11). As with the elaborative-processing hypothesis, forgetting and reconstruction of the action plan are likely to occur in the interval prior to the next movement trial (12).

On the other hand, Schmidt and Wrisberg (2004) divided the practice into two groups: physical practice and mental practice. They believed that mere physical practice does not lead to the proper performance of motor skills, but using mental practice with an imagery format will have a greater impact on performance (13). Mental practice is one of the most important sports psychological interventions that consist of using one or several senses to create or recreate a skill or sports situation (14). Mental practice is considered a recreation of movement patterns in the mind and the most current model of it is imagery (15). Yue (1992), Yaguez (1998), Vielledent (2003), and Mulder (2004) proved that mental practice leads to improvement in motor performance and can have an effect on brain activities during and after mental practice period. In this way, the motor programme is established and the level of muscle activity is mentally regulated (16-19). Bohan et al. (1999) investigated the performance effects of imagery practice at three stages of learning a novel motor task. The results showed that mental practice is most efficient in the first stage of learning. In their study, they proved the relationship between mental practice and experience (20).

Mental practice is affected by some factors, such as task nature-skills that have more cognitive aspects profit from mental practice (21) - and the individual's skill levelprofessional athletes benefit from mental practice significantly (22). There are some theories concerning mental practice. Carpenter (1875) proposed the psychoneuromuscular 
theory and proposed that imagery assists in the learning of motor skills by innervating neuromuscular pathways in a manner similar to when individuals actually performed the activity. When someone vividly imagines an event, the muscles show movement patterns similar to the way they would when actually performing the skill. While these neuromuscular movements are similar to those produced during the actual performance, they are significantly lower in magnitude. Thus, although the magnitude of the muscle activity is reduced during imagery, the activity is a mirror image of the actual performance pattern (23). Sackett (1934) suggested that imagery may function as a coding system to help people understand and gain movement patterns. He argued that imagery can help individuals understand their movements (24). Actions are symbolically coded as a mental map or blueprint (25) and imagery strengthens this blueprint, enabling actions to become more recognizable and automatic. According to this theory, skills that are more cognitive in nature (e.g. kata skill) are more easily coded than pure motor skills (e.g. lifting weights). One way that individuals learn skills is by becoming familiar with that skill and learning what needs to be done in order to become successful. By preparing a motor programme in the central nervous system, a mental blueprint is formed for the successful completion of the movement (26).

There is a challenge associated with the practice programme approach, which relates to the use of physical practice with other practice supplements like mental practice or contextual interference. A significant body of research has been conducted to investigate the effects of CI practices on motor skills. These studies showed that CI practices have different effects on different skills and different situations. Also, the use of CI practice for different ages has different results, as has been explained in last few paragraphs.

On the other hand, mental practice, which is an effective practice supplement, has various impacts on performance and learning motor skills. Many studies that have been conducted in this field showed that the positive effects of mental practice depend on an individual's age and skill level. Considering this theoretical basis and facts, the present study aims to combine CI practice method with mental practice, in order to investigate the results of this combination on the performance and learning of a motor skill. The motor skill that is chosen for this study is the kata skill. As this kind of motor skill has some cognitive aspects, it means that the person who is practising a kata skill has to concentrate and remember movement patterns while performing kata. So, in this skill, the cognitive and movement factors work together at the same time. Therefore, the present study is designed to investigate the effects of mental practice and CI practice on performing and learning a kata skill.

\section{MATERIALS AND METHODS}

Participants. The participants of the research were 36 girls, all elementary school students of Shiraz city (12 years old) who had no prior experience of the task. The sampling method was random. All the participants filled out questionnaires including the individual's information (age, height, weight, etc), medical information (record of illness or pain, mental or muscular diseases), and sports records. The participants completed the MIQ-R questionnaire (Movement Imagery Questionnaire-Revised) (Hall and Martin 1997). This questionnaire consists of eight subscales (four visual imagery, four kinetic imagery). The validity used in this research is criterion validity. The reliability for visual is 0.89 , for kinaesthetic is 0.88 (Atinz et al. 1994), and for the test and re-test is 0.83 (Hall \& Pongrac 1983). The Cronbach's alpha coefficient of the MIQ-R questionnaire in this research is 0.986, which is statistically favourable. The scores were between 40 and 56, and they were randomly placed into one of three groups: (a) mental practice and physical practice with systematic increase in CI (M-CI-P), (b) physical practice with systematic increase in $\mathrm{CI}$ (CI-P), and (c) traditional blocked practice (B$\mathrm{P})$.

The present study consists of three experimental groups, practice sessions, pretest, acquisition, retention, and transfer tests.

Tools. In this research, the first kata, Taikyoku Sono Ichi, was chosen following discussion with the Karate Council and three professional coaches' discretion as the practice programme in the study. This kata consists of 
two-hand and one-foot techniques and rotations (Picture 1).

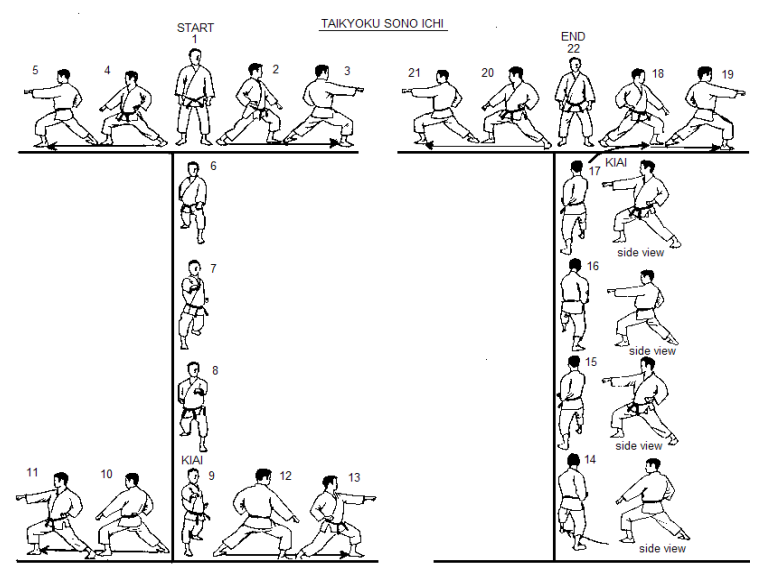

Picture1. Taikyoku Sono Ichi.

Training Protocol. The participants attended the kata pretest after three sessions of physical practice for the initial instruction, and every session was about one hour. Then the groups started their practice schedules. The way of conducting the mental practice was the specific imagination of the kata skill. For the mental practice, the students sat on the ground, closed their eyes, and listened to the instructor. The instructor loudly narrated the movement pattern of the kata skill step by step. The students had to listen to the instructor and imagine that they were performing the movement patterns step by step. Group (a) did the mental practice of the kata skill before commencing physical practices for each effort. So, they practised the kata skill with systematically increasing CI after mental practice of kata for five sessions and five attempts each session. Group (b) practised the kata skill with systematically increasing CI for five sessions and five attempts each session. Group (c) practised the kata skill with the traditional blocked practice for five sessions and five attempts in each session.

Post-tests were taken based on the kata evaluation form. This was taken from the new kata regulation booklet of the World Karate Federation. In this study, the evaluation had been done by an examiner who was an expert karate coach and referee, and the scores were from 0 to 20. The examiner watched the performance of the participants and scored them based on a kata evaluation form. This form consists of some factors such as rhythm, balance, timing, coordination, concentration, techniques, and so on. The acquisition test was in the last training session. In this stage, after group practice, the examiner asked the participants to perform the kata skill one by one and scored them on this. Forty-eight hours after the training session, a retention test was taken and a transfer test was conducted in a competitive condition.

Statistical Analysis. The normality of data was tested using the Shapiro-Wilk test, and the main analysis was performed through a one-way ANOVA for acquisition, retention, and transfer tests. Then Tukey's test was used to compare the difference in the means of the groups by SPSS (version 21) at a significance level of $P \leq 0 / 05$.

\section{RESULTS}

The participants in this study were controlled in indexes such as height, weight, and body composition. The participants of all groups were normal and approximately on the same level (Table 1). The groups' means for the height, weight, and body composition indexes were almost equivalent.

Table1. Demographic Characteristics

\begin{tabular}{ccccc}
\hline group & index & Mean & SD & N \\
\hline \multirow{3}{*}{ CI } & height & 151.50 & 5.95 & \\
& weight & 42.25 & 8.39 & 12 \\
& BC & 18.26 & 2.46 & \\
\hline \multirow{3}{*}{ M-CI } & height & 149.82 & 6.16 & \\
& weight & 43.09 & 6.88 & 11 \\
& BC & 19.24 & 3.18 & \\
\hline \multirow{3}{*}{ B } & height & 150.62 & 5.06 & \\
& weight & 45.23 & 11.47 & 13 \\
& BC & 19.77 & 3.98 & \\
\hline
\end{tabular}

CI: Contextual Interference, M-CI: Mental Practice with Contextual Interference, B: Blocked Practice, BC: Body Composition.

Afterwards, the one-way ANOVA showed no significant difference between the groups in the pretest.

Figure 1 shows descriptive statistics for performance in the acquisition, retention, and transfer tests.

The descriptive one-way ANOVA showed that the B-P group had a better mean than the two other groups in the acquisition test. In the 
retention test, the CI-P group had a better mean than the other groups, and after that the M-CI-P group had a better mean. In the transfer test, results were the same as for the retention test.

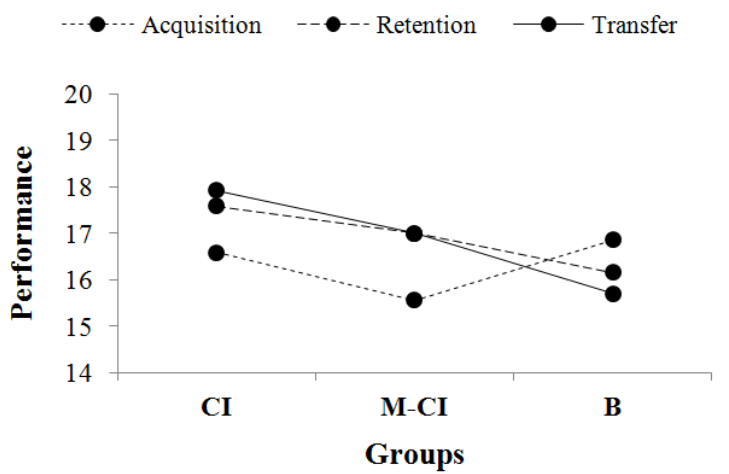

Figure 1. Mean of performance in acquisition, retention and transfer tests. CI: Contextual Interference, M-CI: Mental Practice with Contextual Interference, B: Blocked Practice.

In the next stage, to compare the difference between groups in the acquisition, retention, and transfer tests, a one-way ANOVA analysis was used, as shown in Table 2.

Table 2. One-way ANOVA test of performance

\begin{tabular}{ccccccc}
\hline & & SS & df & MS & F & p \\
\hline \multirow{4}{*}{ Acquisition } & BG & 32.03 & 3 & 10.68 & & \\
& WG & 33.03 & 45 & .73 & 14.55 & .001 \\
& T & 65.06 & 48 & & & \\
\hline \multirow{4}{*}{ Retention } & BG & 17.99 & 3 & 5.99 & & \\
& WG & 39.68 & 45 & .88 & 6.80 & .001 \\
& T & 57.67 & 48 & & & \\
\hline \multirow{4}{*}{ Transfer } & BG & 42.65 & 3 & 14.22 & & \\
& WG & 52.61 & 45 & 1.17 & 12.16 & .001 \\
& T & 95.26 & 48 & & & \\
\hline
\end{tabular}

BG: Between Groups, WG: Within Groups, T: Total, SS: Sum of Squares, MS: Mean Square.

The difference between the groups was significant with $\mathrm{F}=14.55$ and $\mathrm{p}=0 / 001$ in the acquisition, $\mathrm{F}=6.78$ and $\mathrm{p}=0.001$ in the retention, and $\mathrm{F}=12.16$ and $\mathrm{p}=0.001$ in the transfer tests.

Tables 3, 4, and 5 show the difference between the groups with Tukey's test.

The results in the acquisition test revealed a significant difference between the CI-P and MCI-P groups. This shows that the CI-P group was superior, and between M-CI-P and B-P there was a significant difference that shows B$\mathrm{P}$ had a better performance. There was no significant difference between the CI-P and B-P groups in this stage (Table 3 ).

Table 3. Compare means in acquisition

\begin{tabular}{cccccc}
\multirow{2}{*}{ DV } & Group & Group & MD & SE & $\mathrm{p}$ \\
\hline \multirow{3}{*}{ Acquisition } & $\mathrm{CI}$ & $\mathrm{M}-\mathrm{CI}$ & $1.04 *$ & .36 & $* .028$ \\
\cline { 2 - 6 } & $\mathrm{M}$ & $\mathrm{B}$ & -.26 & .34 & .869 \\
\cline { 2 - 6 } & $\mathrm{M}-\mathrm{B}$ & $\mathrm{B}$ & $-1.30 *$ & .35 & $* .003$ \\
\hline
\end{tabular}

DV: Dependent Variable, MD: Mean Difference, SE: Std. Error, CI: Contextual Interference, M-CI: Mental Practice with Contextual Interference, B: Blocked Practice. *: $p<0.05$.

In the retention test, there was a significant difference between the CI-P and B-P groups, and CI-P had a better performance. There were no significant differences between the other groups in this stage (Table 4).

Table 4. Compare means in retention

\begin{tabular}{cccccc}
\hline \multirow{2}{*}{ DV } & \multirow{2}{*}{ Group } & Group & MD & SE & $\mathrm{p}$ \\
\hline \multirow{3}{*}{ Retention } & \multirow{2}{*}{$\mathrm{CI}$} & $\mathrm{M}-\mathrm{CI}$ & .58 & .39 & .453 \\
\cline { 2 - 6 } & & $\mathrm{B}$ & $1.43^{*}$ & .37 & $* .002$ \\
\cline { 2 - 6 } & $\mathrm{M}-\mathrm{CI}$ & $\mathrm{B}$ & .84 & .38 & .139
\end{tabular}

DV: Dependent Variable, MD: Mean Difference, SE: Std. Error, CI: Contextual Interference, M-CI: Mental Practice with Contextual Interference, B: Blocked Practice. *: $\mathrm{p}<0.05$.

In the transfer test, there was a significant difference between the CI-P and B-P groups, which shows CI-P was superior. Also, between M-CI-P and B-P, M-CI-P had a significantly better performance. There was no significant difference between the other groups in this stage (Table 5).

Table 5. Compare means in transfer

\begin{tabular}{cccccc} 
DV & Group & Group & MD & SE & p \\
\hline \multirow{3}{*}{ Transfer } & & & & & \\
\cline { 2 - 6 } & \multirow{2}{*}{ CI } & M-CI & .92 & .45 & .192 \\
\cline { 2 - 6 } & M-CI & B & $1.22 *$ & .43 & $* .001$ \\
\hline
\end{tabular}

DV: Dependent Variable, MD: Mean Difference,

SE: Std. Error, CI: Contextual Interference, M-

CI: Mental Practice with Contextual Interference,

B: Blocked Practice. *: $\mathrm{p}<0.05$. 


\section{DISCUSSION}

There is an important challenge in the use of psychological methods such as mental practice in the field of motor skill training. On the other hand, the use of CI practices along with physical practice had contradictory results in different situations. So, this study combines these two important practice programmes with the aim of comparing the effects of a combined course of mental and physical practice with a systematic increase in CI on the performance of and learning the kata skill.

The results in the acquisition test revealed that the combination of mental practice with CI had no positive effect on the performance of participants, but the blocked practice group had a better performance than the combined group. This part of the results in that phase is in agreement with Sadri et al. (2013). They investigated the effect of $\mathrm{CI}$ on acquisition and learning badminton skills in subjects aged 10-12 years (27). To explain this agreement, the positive effect of a blocked practice in the acquisition stage can be mentioned. According to motor learning theory, researchers often agree that in the first stage of learning a motor skill, there are many cognitive dimensions, most of which are related to a ' What should I do?' question. So, it might not be strange that mental practice, which is the imagination of performing skills, is beneficial for those who are learning a new skill (1). Mental practice can help the individual's response to the questions about performance. But the combination of mental practice with systematically increasing CI showed no positive effect on the performance of the participant in this stage. It might be because participants in this study were novices and could not focus on mental practice and CI at the same time, although it showed long-term positive effects in next stages. Young participants benefit from mental practice in the first stages of learning, but in these stages CI practice has no positive effect on the performance of learners. It might be because of the interference that is happening during the performance. But this interference will be useful in later stages.

From the results in the retention test, it can be deduced that practice with systematically increasing CI had a considerable advantage in the participant's performance after 48 hours in the retention test. It shows that participants memorized task patterns during CI practice and easily remember it after two days. This part of the result is in agreement with the work of Porter and Saemi (2010), who investigated the effect of a systematic increase in CI on three different basketball-related passes. The results showed that practising with gradual increases in CI resulted in a superior performance compared to traditional blocked and random scheduling (6). The results of the present study in this stage can be explained by the elaborative-processing hypothesis that is mentioned in the introduction. According to this hypothesis, the interval prior to the next movement trial is a likely time during which inter-task comparisons are made and the elaboration occurs.

The results in transfer test were considerable. The CI practice group had superior performance compared to the blocked practice group. It shows that practice with systematically increasing CI will have positive effects on the performance in different situations. Also, the M-CI practice group had better performance than the blocked practice group. So, it shows that combination of mental practice with systematically increasing CI will improve performance in various conditions such as competition. Competition is a condition that is different from acquisition and practice stage. The competition context is a stressful situation for participants. Here, they try to perform their best in order to win the competition. The first part of the results in this stage is in agreement with Karimiyani et al. (2013) (28). For skills that consist of different movement patterns (like kata), practice with a systematic increase in CI will have advantages in future attempts. The second part of the results is in agreement with the work of Martin and Hall (1995) (29). The level of skill of the participant is an important factor. Novice learners benefit from the mental practice along with physical practice. It is in agreement with the work of Afsanepurak et al. (2012) (30). The use of a systematic increase in CI will have positive effects on learning motor skills. According to psychoneuromuscular theory, mental practice with activating neuromuscular patterns creates similar effects on muscle function like actual performance (23). So, the use of mental practice with physical practice seems logical to gain the 
best results. Finally, this advantage is explicable with variability in practice. Regular practice repletion leads to discouragement, unwillingness, and disinterest in learners. So, various kinds of practices should come together creatively. This combination may be effective and advantageous due to these facts.

On the other hand, if the task contains many cognitive aspects, it benefits from the mental practice. Mental practice can improve selfconfidence, self-awareness, concentration, and knowledge of task requirements. It can also reduce negative thoughts such as failure and faults (31). The CI practice reconstructionprocessing hypothesis explains that random practice forces the learner to abandon the action plan constructed previously because he or she has to perform a different task on the next trial. The forgetting that ensues requires the learner to actively reconstruct the action plan the next time the initial task is practised, resulting in a stronger memory representation of the practised tasks (11).

\section{CONCLUSION}

Generally, it can be deduced that practice with a systematic increase in CI and a combination of mental practice with CI have a positive effect on the performance and initial learning of a kata skill (Taikyoku Sono Ichi) rather than the traditional blocked practice in novice learners. So, the use of mental practice with a systematic increase in CI can be beneficial for young and novice learners to have effective and favourable performance in various situations such as competition. Considering the fact that this skill includes the cognitive and motor aspects and the learners were 12 years old and novices, it seems that the use of these two types of practice (mental practice and systematic increase in CI) had positive effects on learning the kata skill. But for the definitive conclusion, more detailed research is necessary.

\section{APPLICABLE REMARKS}

- It is recommended that instructors and coaches consider factors such as age, the learner's skill level, and the nature of the skill to provide instruction for developing motor skills, and according to the positive effects of the mentioned practice methods, use them in their practice schedules.

\section{REFERENCES}

1. Magill RA. Motor Learning and Control: Concepts and Applications: McGraw-Hill Higher Education; 2010.

2. Coker CA. Motor learning and control for practitioners: McGraw-Hill Humanities/Social Sciences/Languages; 2004.

3. Magill RA, Hall KG. A review of the contextual interference effect in motor skill acquisition. Human movement science. 1990;9(3):241-89.

4. Porter JM. Systematically increasing contextual interference is beneficial for learning novel motor skills: Faculty of the Louisiana State University and Agricultural and Mechanical College in partial fulfillment of the requirements for the degree of Doctor of Philosophy in The Department of Kinesiology by Jared Marak Porter BS, University of Central Missouri; 2008.

5. Porter J.M, \& Magill R.A. Systematically increasing contextual interference is beneficial for learning sport skills. 2010; 28(12), 1277-1285.

6. Porter J.M, \& Saemi E. Moderately skilled learners benefit by practicing with systematic increases in contextual interference. International journal of coaching science. 2010; 4(2), 61-71.

7. Saemi E, Porter J.M, GotbiVarzaneh A, Zarghami M, \& Shafinia P. Practicing along the contextual interference continuum: a comparison of three practice schedule in an elementary physical education setting. Kineziologija. 2012; 44(2), 191-198.

8. Shea J.B, \& Morgan R.L. Contextual interference effects on the acquisition, retention, and transfer of a motor skill. Journal of experimental psychology: human learning and memory. 1979; 5(2), 179

9. Shea J.B, \& Titers R.C. Journal o motor behavior.1993;25, 264-274.

10. Shea J.B, \& Zimny S.T. Context effects in memeory and learning movement information. In R.A. Magil (Ed), memory and control of action. Amsterdam: North-Holland.1983;Pp. 345-366.

11. Lee T.D, \& Magill R.A. The focus of contextual interference in motor skill acquisition. Journal of experimental psychology: learning memory and cognition. 1983;9(4), 730. 
12. Lin C. H, Fisher B. E, Winstein C. J, Wu A. D, \& Gordon J. Contextual Interference Effect: Elaborative Processing or Forgetting - Reconstruction? A Post Hoc Analysis of Transcranial Magnetic Stimulation —-Induced Effects on Motor Learning. Journal of motor behavior. 2008;40(6), 578-586.

13. Schmidt R.A, \& Wisberg C. Motor learning and performance. $3^{\text {rd }}$ ed. Human kinetics. 2004.

14. White A, Hardy L. Use of different imagery perspectives on the learning and performance of different motor skills. British psychology.1995;86,2, 191-216

15. Mc Morrise T. Acquisition \& performance of sport skill. 2007.

16. Yue G, \& Cole K.J. Strength increases from the motor program: comparison of training with maximal voluntary and imagined muscle contractions. J neurophysiol. 1992;67: 1114-1123.

17. Yaguez L, Nagel D, Hoffman H, Canavan A.G, Wist E, \& Homberg V.A. Mental route to motor learning: improving trajectorial kinematics theough imagery training. Behave brain. 1998;90(1): 95-106.

18. Vielledent S, Kosslyn S.M, Berthoz A, \& Giraudo M.D. Does mental stimulation of following a path improve navigatin performance without vision? Cogn brain. 2003;16(2), 138-249.

19. Mulder T, Ziglstra S, Ziylstra W, \& Hochstenbach J. The role of motor imagery in learning a totally novel movement. Exp Brain. 2004;154(2): 211-217.

20. Bohan M, Pharmer JA, \& Stokes AF. When does imagery practice enhance performance on a motor task? Perceptual and motor skills. 1999;88(2), 651-658.

21. Felt D, Landers D, \& Becker M. The effect of mental practice on motor skill learning and performance: A metaanalysis, Journal of Sport psychology. 1983;5: 25-57.

22. Hall C. Imagery in sport and exercise. Handbook of sport psychology. 2001;2:529-49.

23. Carpenter WB. Principles of mental physiology: With their applications to the training and discipline of the mind, and the study of its morbid conditions: HS King \& Company; 1875.

24. Sackett RS. The influence of symbolic rehearsal upon the retention of a maze habit. The Journal of General Psychology. 1934;10(2):376-98.

25. Vealey R. S, \& Walter S. M. Imagery training for performance enhancement and personal growth. In J. M. Williams (Ed.), Applied sport psychology: Personal growth to peak performance. 1993; (2nd ed., pp. 200-224). Mountain View, CA: Mayfield.

26. Kim B. H., \& Giacobbi, P. R. (2009). The Use of exercise-related mental imagery by middle-aged adults. Journal of imagery research in sport and physical activity, 4(1).

27. Sadri, K, Mohammadzadeh H, \& Khani M. The effect of contextual interference on acquisition and learning a badminton skill among children aged from 10 to 12. Annals of applied sport science. 2013;1(3), 39-46.

28. Karimiyani N, Sami S, Hakimi M, Ali Mohammadi M, \& Mahmoudi S. The effect of blocked, random and systematically increasing practice schedule on learning of dart-throwing skill. Annual of biological research.2013;4(11): 129-133.

29. Martin K, \& Hall C. Using mental imagery to enhance intrinsic motivation. The journal of sport \& exercise psychology. 1995; 17, 54-69.

30. Afsanepurak S. A, Karimiani N, Moradi J, \& Safai M. The effect of blocked, random and systematically increasing practice on learning of different types of basketball passes. European journal of experimental biology. 2012;2(6), 2397-2402. 